\title{
MR imaging findings of germ cell tumors arising from the basal ganglia: focused on early imaging finding
}

\author{
So Mi Lee ${ }^{1,2}$, In-One Kim
}

'Department of Radiology, Seoul National University College of Medicine, Seoul, Korea

${ }^{2}$ Department of Radiology, Kyungpook National University Medical Center, Daegu, Korea

Article Info

\section{Article Notes}

Received: July 06, 2016

Accepted: July 12, 2016

\section{${ }^{*}$ Correspondence:}

Dr. In-One Kim

Department of Radiology and Institute of Radiation Medicine,

Seoul National University College of Medicine , 101 Daehak-ro,

Jongno-gu, Seoul 110-769, Republic of Korea

Telephone: +82-2-2072-3608

Fax: +82-2-747-5781

Email: kimio@snu.ac.kr

(c) $2016 \mathrm{Kim} \mathrm{IO}$. This article is distributed under the terms of the Creative Commons Attribution 4.0 International License

\section{Keywords}

Germ cell tumor

Basal ganglia

Early stage

Serial image

MRI

\section{ABSTRACT}

Germ cell tumor (GCT) arising in the basal ganglia or thalami is relatively uncommon. It occurs most commonly in boys during second decade of life. It is difficult to diagnose early stage GCTs in these regions because the images are not so typical and the symptom onset is insidious. However, early diagnosis of this tumor is important because of the high radiosensitivity and potential curability. Early stage GCTs originating from the basal ganglia or thalami appear as ill-defined small patchy lesions. They frequently present as hyperintense lesions compared with deep gray matter on T2-weighted image without cyst, mass effect, or prominent enhancement. Microhemorrhages can be accompanied infrequently. These tumors are mostly associated with ipsilateral hemiatrophy at the time of presentation. During tumor progression, tiny cysts develop at a relatively early stage, and intratumoral cyst, hemorrhage, and ipsilateral hemiatrophy gradually tend to be more pronounced. Ultimately, these become overt large mass with remarkable heterogenous enhancement, containing multiple cysts of various sizes and hemorrhage. This review aims to describe the serial MR imaging findings of the GCTs arising from basal ganglia or thalami, focusing on the early finding.

\section{Introduction}

Intracranial germ cell tumor (GCT) commonly originates in the pineal or suprasellar regions, but a significant portion of the tumors, estimated at $4 \%$ to $14 \%$, arise in the basal ganglia or thalami, particularly in young males ${ }^{1-3}$. Based on histopathology, GCTs are classified into germinoma and non-germinomatous GCTs ${ }^{3}$. GCT arising from basal ganglia or thalami have special concerns with regard to early diagnosis due to atypical presentation and insidious clinical course: patients with GCT arising from these locations frequently present with only vague symptom such as decreased school performance and mild cognitive disturbance in the early stage ${ }^{2-5}$. Neuroimaging findings in the early stage are often subtle [6]. Therefore, the diagnosis may be challenging and can be delayed until an advanced stage of disease is reached. As GCTs, especially germinomas, are relatively radiosensitive and potentially curable, early diagnosis is essential for better outcome $\mathrm{e}^{2-3}$. The purpose of this review is to discuss the serial MR imaging findings of the GCTs arising from basal ganglia or thalami, focusing on the early finding.

\section{Early MR Imaging Finding}

Radiologic characteristics are similar between germinoma and 
non-germinomatous $\mathrm{GCTs}^{3}$, and most of the previous reports have been discussed regarding neuroimaging finding of the germinoma. On magnetic resonance imaging (MRI), early stage of germinomas usually appears as ill-defined, homogenous, patchy solid lesion, without mass effect ${ }^{7-9}$. The tumors are generally isointense to hyperintense compared with cortical gray matter on $\mathrm{T} 2$ weighted, T1-weighted, and FLAIR images, owing to the high cellularity and relatively low water contents ${ }^{1}$. Small tumors may appear as slightly hyperintense lesion relative to the basal ganglia on T2-weighted image, as a result of iron deposition in the globi pallidi during adolescence. Contrast enhancement is not prominent in the early stage. The majority of germinomas predominantly show normal to restricted diffusion ${ }^{10}$. Indeed, the conventional MR imaging finding of the germinoma itself can be subtle and inconspicuous in the early stage.

Recently, susceptibility-weighted imaging has been proven as a useful technique for diagnosis of early basal ganglia germinoma $a^{6,711}$. Small tumor at early stage frequently appears as obvious hypointense lesion on susceptibility-weighted imaging owing to the characteristic intratumoral hemorrhage and tumor induced metal accumulation, even when it was indistinct on conventional imaging ${ }^{7,12}$. However, hypointensity on susceptibilityweighted imaging is relatively not remarkable in the nontumorous condition such as infarction ${ }^{11}$. Therefore, susceptibility-weighted imaging could be helpful in differential diagnosis as well as in early detection of basal ganglia germinoma.

Hemiatrophy in the ipsilateral hemisphere and brainstem, which is frequently present at the time of presentation, is also useful for diagnosing basal ganglia GCTs $^{8,9,13}$. This feature was thought to be characteristic of germinoma arising from the basal ganglia or thalami because of common association with this tumor. However, ipsilateral hemispheric and brainstem atrophy is now regarded as a result of involvement of internal capsule or thalamic ganglion cell by any kind of brain pathology ${ }^{14}$. Germinomas, showing irregular margin, are frequently involve the internal capsule or periphery of the thalamus even in the small tumor. This tumor location may be a reasonable explanation for common association with ipsilateral hemispheric and brainstem atrophy in the basal ganglia germinoma. In contrast, relatively well marginated tumor restricted to the basal ganglia may not be associated with this feature ${ }^{6,9}$. Ipsilateral hemispheric and brainstem hemiatrophy also may be influenced by slow growth rate of the germinoma in addition to tumor location. Like germinoma, other slowly growing tumor could also develop ipsilateral hemiatrophy in the hemisphere and brainstem, while this feature is very rare for a rapid growing tumor ${ }^{13,15}$.

\section{Serial MR Imaging Change during Tumor Progression}

The indolent nature and atypical symptom of the GCTs in the basal ganglia or thalami may lead to long-term latency before diagnosis and render a timely diagnosis difficult ${ }^{5,6}$. In some case in which the diagnosis is equivocal or inconclusive, follow-up examination may be performed. A significant number of basal ganglia GCTs stay for up to several years, and it accordingly could be misinterpreted as non-tumorous condition ${ }^{11}$. Therefore, knowledge about sequential neuroimaging findings of this tumor could allow radiologists to diagnose this tumor correctly.

During follow-up period, intratumoral tiny cyst may develop in the ill-defined patchy small tumor, although overall tumor size is not significantly changed. Multiple intratumoral cysts of various size are typically found in late-stage basal ganglia GCTs, however tiny cysts can occur at the relatively early stage during tumor progression ${ }^{6}$. The majority of the cysts have fluid-fluid level with markedly hypointense dependant layering or dark-intensity rim on T2-weighted image, indicating repeated intratumoral hemorrhage ${ }^{6}$. At initial MR imaging, tumor presenting as a small basal ganglia mass containing a few tiny cysts can be mistaken for a nontumorous condition such as vasculitis, cavernous malformation, or cerebral infarction. However, basal ganglia GCT should be considered as one of differential diagnosis based on such a progression process, especially in boys. Cyst may develop as a result of necrosis or repeated intratumoral hemorrhage, and cystic change and hemorrhage seems to be attributable to tumor enlargement ${ }^{16-18}$. Changes in sequential feature of the basal ganglia GCTs support this theory even further ${ }^{6}[6]$. As tumor grows, cystic change and hemorrhage within tumor are generally more obvious: the cysts not only increase in size but also occupy a greater portion of the tumor, moreover intra-cystic hemorrhage is usually more pronounced during tumor progression ${ }^{6}$. The overt large mass ultimately contains multiple cysts of various sizes and hemorrhage. The timescale for tumor progression is variable in each tumor, and it can take for more than several years ${ }^{6}$.

Hemiatrophy in the ipsilateral hemisphere and brainstem, which is frequently visible at the time of presentation, is generally more pronounced during tumor progression. As it is considered as a result of Wallerian degeneration caused by tumor infiltration of fiber tracts, tumor enlargement may naturally lead to progression of ipsilateral hemiatrophy. Interestingly, ipsilateral hemispheric and brainstem atrophy can progress, although tumor size is not changed during follow up ${ }^{6}$.

Tumor enhancement also becomes more prominent and heterogenous, as tumors progress ${ }^{6,19}$. Small patchy lesion without mass effect and cystic component usually shows faint or no contrast enhancement. As tumor grows, mild nodular enhancement may occur in some portion 
of the tumor. The overt large mass containing multiple cysts and hemorrhage generally shows remarkable and heterogenous enhancement. (Figure1)

\section{Late MR Imaging Finding}

Late stage of the GCTs arising from basal ganglia or thalami usually appear as overt large masses which consist of solid portion, various cystic component, and hemorrhage $\mathrm{e}^{1,18,20}$. Heterogeneity is common in GCTs arising in this regions, unlike that of the suprasellar or pineal regions: most of the tumors more than $2-3 \mathrm{~cm}$ in diameter contain hemorrhage as well as cyst, furthermore some degree of necrosis is present in $80 \%(50 \%$ are slightly necrotic, $30 \%$ are mostly necrotic) at the time of presentation $^{1,6,17,18,20,21}$. GCTs of this regions show relatively less mass effect and peritumoral edema, therefore tend to be large at the time of presentation ${ }^{1,18}$. The solid portion is generally isointense relative to the signal intensity of the cerebral cortex and usually markedly enhance after contrast infusion. At this time, presence of accompanying cerebrospinal fluid-borne metastases in the brain and spine, which shows also remarkable enhancement, should be considered, because it may be associated with tumor progression ${ }^{19}$. When large mass in the basal ganglia or thalami is isointense to the cerebral cortex, particularly in assoication with cysts, hemorrhage, or ipsilateral hemiatrophy, GCTs should be considered, along with lymphoma and primitive neuroectodermal tumor. Prevalence in the young male predominance is helpful in the differential diagnosis.

\section{Added Value of PET in the Diagnosis of GCTs}

Basal ganglia germinomas generally show discernible uptake in 11C-methionine-PET in contrast, hypometabolism in 18F-FDG-PET ${ }^{22,23}$. These metabolic imaging using amino acid or glucose can be helpful in

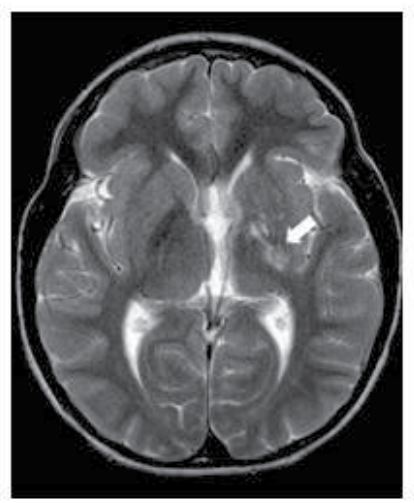

A

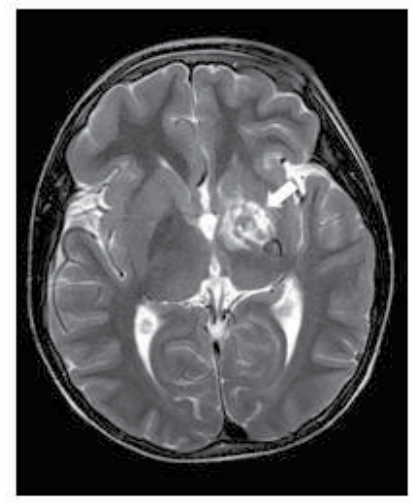

D

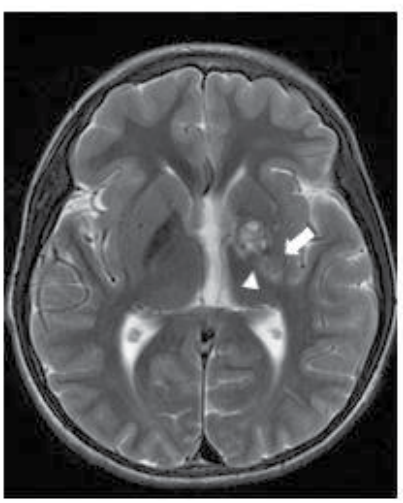

B

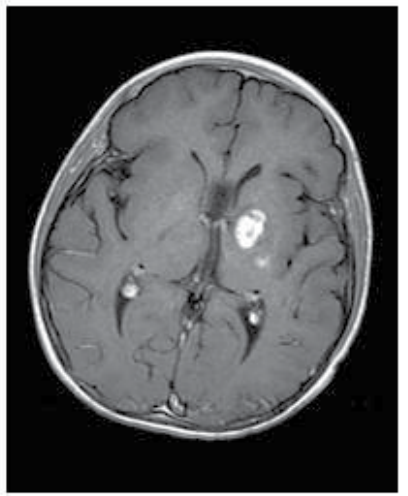

E

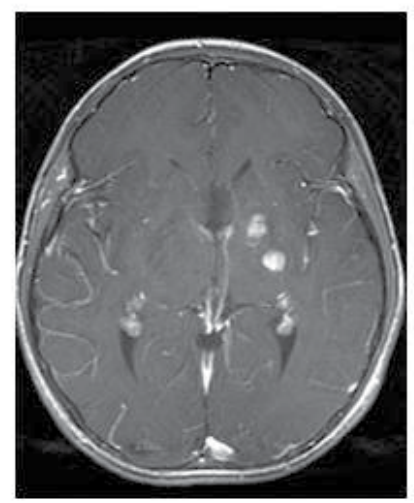

C

Figure 1: An 11-year-old boy with mixed GCT in the left basal ganglia. A-E. Axial T2-weighted and contrast enhanced images illustrate a staged change during tumor progression. A. Initial T2-weighted image shows an ill-defined hyperintense lesion without evident cystic component or mass effect in the lentiform nucleus and internal capsule (arrow). Ipsilateral hemiatrophy is seen in the BG and thalamus. (B-C): B. Two months after initial MRI, tumor (arrow) shows cystic change and increased size. Tiny cysts have fluid-fluid level with marked hypointensity in the dependant portion suggesting hemorrhage (arrowhead). C. Post contrast T1 WI shows marked enhancement. (DE): Seven months after initial MRI. D. Tumor (arrow) has changed to an overt large mass contining multiple cysts of various sizes and hemorrhage E. Tumor shows rather homogenous solid enhancement. 
differential diagnosis. Hypometabolism on 18F-FDG-PET enables differentiation of germinoma from other highgrade tumors such as lymphoma, high-grade glioma, and primitive neuroectodermal tumor, while this feature may make it difficult to distinguish germinoma from nontumorous condition such as cerebral infarction. 11C-methionine-PET have an advantage of differentiating tumor from nontumorous condition: the demand of amino acid is increased during malignant transformation unlike nontumorous condition, in addition it is less influenced by inflammation than the 18F-FDG-PET ${ }^{23}$. 11C-methioninePET has a high diagnostic value for detecting basal ganglia germinoma, therefore can aid earlier diagnosis ${ }^{19,22,23}$.

\section{Conclusion}

Here, we review the serial MR imaging findings of GCTs arising basal ganglia or thalami, focused on early finding. For earlier detection of basal ganglia or thalami GCTs, not only high radiologic suspicion but also active diagnostic procedures aided by $11 \mathrm{C}$-methionine-PET are required.

\section{References}

1. Raybaud C, Barkovich AJ. Intracranial, orbital, and neck masses of childhood in Pediatric Neuroimaging. Barkovich AJ, RaybaudC, Editors. 2012, Lippincott Williams \& Wilkins, 2012: Philadelphia: Wolters Kluwer Health.

2. Packer RJ, Cohen BH, Cooney K. Intracranial germ cell tumors. Oncologist. 2000; 5(4): 312-20.

3. Echevarría ME, Fangusaro J, Goldman S. Pediatric central nervous system germ cell tumors: a review. Oncologist. 2008;13(6):690-9.

4. Wataya T, Ishizaki R, Kitagawa M, Tashiro Y. Germinoma in the bilateral basal ganglia presented with cognitive deterioration. Childs Nerv Syst. 2015;31(6):953-8.

5. Phi JH, Kim SK, Lee YA, et al. Latency of intracranial germ cell tumors and diagnosis delay. Childs Nerv Syst. 2013;29(10):1871-81.

6. Lee SM, Kim IO, Choi YH, et al. Early imaging findings in germ cell tumors arising from the basal ganglia. Pediatr Radiol. 2016;46(5):71926.

7. Lou X, Ma L, Wang FL, et al. Susceptibility-weighted imaging in the diagnosis of early basal ganglia germinoma. AJNR Am J Neuroradiol. 2009;30(9):1694-9.

8. Ozelame RV, Shroff M, Wood B, et al. Basal ganglia germinoma in children with associated ipsilateral cerebral and brain stem hemiatrophy. Pediatr Radiol. 2006;36(4):325-30.
9. Okamoto K, Ito J, Ishikawa $\mathrm{K}$, et al. Atrophy of the basal ganglia as the initial diagnostic sign of germinoma in the basal ganglia. Neuroradiology. 2002;44(5):389-94.

10.Douglas-akinwande AC, Ying J, Momin Z, Mourad A, Hattab EM. Diffusion-weighted imaging characteristics of primary central nervous system germinoma with histopathologic correlation: a retrospective study. Acad Radiol. 2009;16(11):1356-65.

11.Lou X, Tian C, Chen Z, Ma L. Differential diagnosis of infarct-like intracranial ectopic germinomas and subacute lacunar infarct on susceptibility-weighted imaging. J Magn Reson Imaging. 2012;36(1):92-8.

12. Rouault TA, Cooperman S. Brain iron metabolism. Semin Pediatr Neurol. 2006; 13: 142-8.

13. Wong ST, Yuen SC, Fong D. Pathophysiological mechanism of ipsilateral cerebral and brainstem hemiatrophy in basal ganglia germ cell tumors: case report. Childs Nerv Syst. 2009;25(6):693-9.

14.Kuhn MJ, Mikulis DJ, Ayoub DM. Wallerian degeneration: evaluation with MR imaging. Radiology. 1988; 168: 199-202.

15. Mutoh $\mathrm{K}$, Okuno $\mathrm{T}$, Ito $\mathrm{M}$, et al. Ipsilateral atrophy in children with hemispheric cerebral tumors: CT findings. J Comput Assist Tomogr. 1988;12(5):740-3.

16. Soejima T, Takeshita I, Yamamoto H, Tsukamoto Y, Fukui M, Matsuoka S. Computed tomography of germinomas in basal ganglia and thalamus. Neuroradiology. 1987;29(4):366-70.

17. Higano S, Takahashi S, Ishii K, Matsumoto K, Ikeda H, Sakamoto K. Germinoma originating in the basal ganglia and thalamus: MR and CT evaluation. AJNR Am J Neuroradiol. 1994;15(8):1435-41.

18. Moon WK, Chang KH, Kim IO. Germinomas of the basal ganglia and thalamus: MR findings and a comparison between MR and CT. AJR Am J Roentgenol. 1994; 162: 1413-7.

19. Phi JH, Cho BK, Kim SK, et al. Germinomas in the basal ganglia: magnetic resonance imaging classification and the prognosis. J Neurooncol. 2010;99(2):227-36.

20. Kim DI, Yoon PH, Ryu YH, Jeon P, Hwang GJ. MRI of germinomas arising from the basal ganglia and thalamus. Neuroradiology. 1998;40(8):50711.

21. Rasalkar DD, Chu WC, Cheng FW, Paunipagar BK, Shing MK, Li CK. Atypical location of germinoma in basal ganglia in adolescents: radiological features and treatment outcomes. $\mathrm{Br} J$ Radiol. 2010;83(987):261-7.

22.Sudo A, Shiga T, Okajima M, et al. High uptake on 11C-methionine positron emission tomographic scan of basal ganglia germinoma with cerebral hemiatrophy. AJNR Am J Neuroradiol. 2003;24(9):1909-11.

23. Lee J, Lee $\mathrm{BL}$, Yoo $\mathrm{KH}$, et al. Atypical basal ganglia germinoma presenting as cerebral hemiatrophy: diagnosis and follow-up with 11C-methionine positron emission tomography. Childs Nerv Syst. 2009;25(1):29-37. 